BRIEF REPORT

\title{
Communicating information on cardiopulmonary resuscitation to hospitalised patients
}

\author{
R Sivakumar, J Knight, C Devlin, P Keir, P Ghosh, S Khan
}

J Med Ethics 2004;30:311-312. doi: 10.1136/jme.2002.002915

Aim: The primary aim of the study was to evaluate two different methods of communicating information on cardiopulmonary resuscitation (CPR) to patients admitted to general medical and elderly care wards. The information was either in the form of a detailed information leaflet (appendix I) or a summary document (appendix II). The study examined the willingness of patients in seeking detailed information on cardiopulmonary issues.

Setting: The study was conducted over three months on a general medical ward and an acute elderly care ward in two district general hospitals.

Methods: A detailed information leaflet on CPR was provided to the nursing staff on the wards. An A4 summary document summarising the CPR decision making process and basic information on cardiopulmonary issues was placed in a folder at the foot of each bed on the elderly care ward. On the general medical ward it was displayed prominently over the head of all beds.

Results: Out of the 274 patients admitted to the general medical ward only two requests were received for the detailed information leaflet. On the elderly care ward there were 182 admissions but no patients or their relatives requested the leaflet.

Conclusions: Availability of basic information on cardiopulmonary resuscitation to all patients is practical and does not lead to unnecessary distress or offence to patients or their carers. It makes the decision making process more transparent. Detailed information leaflets are of value for a minority of hospitalised patients.

$\mathrm{E}$ ffective communication with patients and their carers is the most important issue surrounding cardiopulmonary resuscitation (CPR). The need to develop sensitive and reliable ways to improve communication and help patients make valid choices cannot be overemphasised.

For the majority of hospitalised patients CPR is an unlikely event, and hence provision of detailed information to every patient particularly when not requested would be inappropriate and could be misinterpreted. ${ }^{1}$ The emphasis should be on giving patients the opportunity to be involved in end of life decisions rather than to compel them to become involved or make choices.

There is evidence that few patients actually want to be involved in final resuscitation decision making. ${ }^{2}$ There is also evidence that a significant proportion of medical professionals have concerns regarding initiating discussion relating to resuscitation issues. ${ }^{3}$ High demand on doctor and nurse time makes it even more difficult for patients to acquire detailed information and make informed choices.

We explored two methods of communicating sensitive information on CPR to hospitalised patients and their carers.
Our aim was to offer information in a humane manner to those who were interested but to avoid imposing on patients who were not keen to know.

\section{DESIGN AND METHODS}

The study was approved by the Trust's Resuscitation Committee and the Board of Clinical Practice. Comments were also invited from the Trust's ethics committee.

The study was conducted over a three month period from June to September 2002. All patients admitted to a designated general medical ward and an acute elderly care ward were included.

The detailed information leaflet (appendix I; please visit www.jmedethics.com/supplemental) contained literature on most aspects of CPR which could be of use to the patients or their carers, and was kept by the nursing staff on both wards with a view to providing it to patients or their carers on request. Records were kept regarding handing out this information and written feedback was requested in the form of a questionnaire survey. The completed questionnaire was sent to the audit department.

The summary document (appendix II; please visit www.jmedethics.com/supplemental) was produced in large print and was laminated and provided basic information on CPR. It encouraged the reader to seek more information if they desired and to acquire the detailed information leaflet, which was also available in larger print for people with impaired vision.

On the elderly care ward the summary document was placed at the foot of each bed. This was the first document within a folder. It was available to patients and their carers if they wished to read it. On the medical ward the summary document was pasted in a prominent position over the head of each bed.

Training was organised for the nursing staff on both wards before the study. Records were kept for any concerns or complaints expressed by the patients, their relatives, or indeed any member of the staff.

\section{RESULTS}

During the three months 456 patients were admitted to the two wards. Out of the 274 patients admitted to the general medical ward only two requests were received for the information leaflet. Those who sought this information found it to be very useful. No requests for information leaflets were received from the relatives.

On the elderly care ward there were 182 admissions but no patients or their relatives requested the information leaflet.

No concerns were expressed by the patients, their relatives, or carers regarding any aspect of the study. The only comment received from one staff member was to consider changing the colour of the leaflets, as the colour purple was believed to be associated with death.

Abbreviation: CPR, cardiopulmonary resuscitation. 


\section{DISCUSSION}

There is a general consensus on identifying patients not suitable for CPR, thus ensuring dignity at the end of life. However, controversy surrounds discussions on CPR with every intellectually competent patient. There are some who favour frank discussion with every patient respecting the patient's autonomy ${ }^{4}$ while others do not approve of such discussions if the "not to resuscitate" decision is made on the grounds of futility. ${ }^{5}$ The advocates of the latter point out that failing to take into account the emotional impact of such a discussion could be devastating for a frail and ill patient. This could even be construed as being inhumane at a time of maximal emotional vulnerability. The psychological pain caused by such discussions with patients and the resulting uneasiness among medical professionals is well recognised. ${ }^{3}{ }^{6}$

Another fundamental question is whether all patients are interested in the CPR decision making process. The literature shows wide variation in reported percentage of interested patients. Some studies have interviewed patients who were about to be discharged from hospitals. Others used outpatient settings and therefore were not truly representative of hospitalised patients with significant pathology in whom CPR is a likely outcome.

The joint statement from the British Medical Association, the Resuscitation Council (UK) and the Royal College of Nursing suggested that written information about resuscitation policies should be included in the general literature provided to patients in hospitals and patients should be encouraged to see such information. ${ }^{7}$ As cardiorespiratory arrest is a rare event for the majority of hospitalised patients, provision of detailed information to each patient would neither be practical nor appropriate.

Rather than providing detailed information to every patient, we explored the use of a summary document (appendix II) and found it to be a practical way of communicating basic CPR information. This method did not generate anxiety or complaints and helped in making the decision making process more transparent for patients and their carers. During the study period, no formal requests were made by patients or their relatives for discussions with medical teams.

The detailed information leaflet was available only for those who wished to acquire such information. The patients who desired this material were expecting explicit information on end of life issues and therefore were neither disturbed nor offended by it. Although rated to be useful by those who requested these leaflets, their uptake was low.

We also explored two different methods of offering basic CPR information to patients on the wards. The medical ward had the summary document pasted above the head of each bed. Despite the document being in a prominent position, in our experience this did not subject the patients or their relatives to unnecessary anxiety.

Our study had a few inherent weaknesses. Although efforts were made to place the summary document in a prominent position on each ward, it was not recorded whether every patient or their carer had the opportunity to examine this information. However, any attempt to do so may have inadvertently compelled some patients to discuss CPR issues against their wishes. The other weakness of this study was the inability to identify the proportion of patients who were incompetent and/or were unable to read. The results showed that the overwhelming majority of the patients or their relatives did not initiate discussion about CPR, a trend seen in published literature. ${ }^{8}$ However, the design of this study cannot determine the precise reason for this trend. Further research is needed to elucidate the reasons for low uptake of detailed information on CPR. Feedback on information leaflets from patients, their carers, and relatives would be desirable in improving communication on end of life issues.

\section{CONCLUSIONS}

We conclude that availability of basic information on cardiopulmonary resuscitation to all patients is practical and does not lead to unnecessary distress or offence to patients or their carers. It makes the decision making process more transparent and helps improve the patient's confidence. Detailed information leaflets are of value for a minority of hospitalised patients.

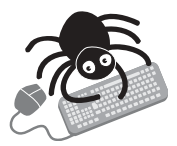

The appendices can be viewed on the JME website (www.jmedethics.com/supplemental)

\section{Authors' affiliations}

R Sivakumar, P Ghosh, S Khan, Department of Elderly Care, Lister Hospital, Stevenage, UK

J Knight, C Devlin, East and North Hertfordshire NHS Trust, UK

P Keir, Department of Cardiology, Queen Elizabeth II Hospital, Welwyn Garden City, UK

Correspondence to: Dr R Sivakumar, Strathmore Wing, Lister Hospital, Coreys Mill Lane, Stevenage, UK; sivasiva51@hotmail.com

Received 25 December 2002

Revised version received 30 March 2003

Accepted for publication 2 June 2003

\section{REFERENCES}

1 Heller A, Potter J, Sturgess I, et al. Resuscitation and patients' views: Questioning may be misunderstood by some patients. BMJ 1994;309:408.

2 Florin D. Decisions about cardiopulmonary resuscitation. BMJ 1994:308:1653-4.

3 Schade SG, Muslin H. Do not resuscitate decisions: discussions with patients. J Med Ethics 1989; 15:186-90.

4 Ebrahim S. Do not resuscitate decisions: flogging dead horses or a dignified death? BMJ 2000;320:1155-6.

5 Stewart K. Discussing cardiopulmonary resuscitation with patients and relatives. Postgrad Med J 1995;71:585-9.

6 Stolman CI, Gregory JJ, Dunn D, et al. Evaluation of patient, physician, nurse and family attitudes toward do not resuscitate orders. Arch Intern Med 1990;150:653-8.

7 Decisions relating to Cardiopulmonary resuscitation. A joint statement from the British Medical Association, the Resuscitation Council (UK) and the Royal College of Nursing. http://www.resus.org.uk/pages/dnar.htm (accessed 10 October 2002).

8 Stewart K, Spice C. Not discussing decisions is often because of practicalities, not ageism. BMJ 2001;322:102. 